\title{
Análisis del estado actual de apertura de datos en salud a nivel autonómico a través de los portales de datos abiertos
}

Health information opennes in Spanish regional governments' open data portals

\section{María Ángeles Bertos QUesAdA (1), Gonzalo MARCo CuencA (2)}

(1) Documentalista. Facultad de Filosofía y Letras. Universidad de Zaragoza. (2) Departamento de Ciencias de la Documentación e Historia de la Ciencia. Facultad de Filosofía y Letras. Universidad de Zaragoza, gmarco@unizar.es

\begin{abstract}
Resumen
Se analiza el nivel de apertura de datos de las Comunidades Autónomas en España a través de sus portales de datos, centrando el análisis en el estado actual de publicación de datos abiertos en materia de salud. Para ello, se han desarrollado un total de diez indicadores que han sido organizados por dominios. Han sido publicados un total de 485 conjuntos de datos de salud, que equivalen al $2,5 \%$ del total de los conjuntos de datos. Las estadísticas sanitarias son los datos que más se publican en la categoría. El $62,4 \%$ de las comunidades autónomas publica en formatos reutilizables y no propietarios. Este análisis refleja una publicación a varias velocidades y la existencia de unas comunidades autónomas más adelantadas que otras en la publicación de este tipo de datos.
\end{abstract}

Palabras clave: Datos abiertos. Portales de datos abiertos. Datos abiertos en salud. Reutilización de la información. España.

\section{Introducción}

Los datos pueden ser considerados como elementos básicos en el registro de eventos o actividades. El dato, como tal, es un contenido que alimenta un contexto específico. Por ejemplo, un código postal, una fecha o el identificador de un libro son datos que, sin un propósito, una utilidad o un contexto, no sirven de mucho. Sin embargo, la ingesta de estos datos dentro de un contexto preciso, formando conjuntos de datos diferenciados, puede ser muy relevante.

Las Administraciones Públicas, en el ejercicio de sus funciones, generan una gran cantidad de conjuntos de datos o de información, pagada con el dinero de los ciudadanos, que es susceptible de ser reutilizada (Pintos y Marín, 2013, p. 15). Estos conjuntos de datos representan activos de información que pueden ser compartidos y reutilizados por terceras personas si los distribuimos en formatos abiertos. En este sentido, las iniciativas de datos abiertos se constituyen como un

\begin{abstract}
The level of data openness of the autonomous regions of Spain through their open data portals is researched, focusing the analysis on the current state of publication of open data on health. For this, a total of ten indicators have been developed and organized by domains. A total of 485 health data sets have been published, equivalent to $2.5 \%$ of all data sets. Health statistics are the most widely published data in the category. The $62.4 \%$ of the autonomous regions publish in reusable and non-proprietary formats. This analysis reflects a publication at various speeds and the existence of some autonomous regions more advanced than others in the publication of this type of data.
\end{abstract}

Keywords: Open data. Open data portals. Health information. Information reuse. Spain.

elemento clave que favorece las políticas de transparencia de las Administraciones Públicas, aseguran el libre acceso a los datos a todos los ciudadanos y promueven oportunidades de negocio a partir de la reutilización de los datos como materia prima (Arquero Avilés y Marco Cuenca, 2014 , p. 100). Para que los datos abiertos de las administraciones públicas sean fáciles de usar y puedan ser considerados realmente como un activo de información de calidad, deberían ser datos: actualizados, objetivos, útiles y accesibles (Cerrillo, 2014, p. 70).

Los beneficios específicos que podemos obtener de la liberación de datos dependerán, en gran medida, de la propia calidad de los datos y, sobre todo, de la capacidad de reutilizarlos para obtener valor. Sin embargo, los datos abiertos no suelen encontrarse siempre de este modo, en muchas ocasiones se encuentran incompletos, desactualizados, fragmentados, mal clasificados $y$, consecuentemente, pueden ser considerados de baja calidad. En este sentido, Sadiq e Indulska 
(2017, p. 150) consideran que uno de los mayores riesgos relacionados con el uso de datos abiertos es la falta de conocimiento de la calidad inherente de los datos, ya que frecuentemente se usan para un propósito que originalmente no estaba previsto en el momento de su recopilación $y$, por tanto, podrían ser de calidad insuficiente o no aptos para otros propósitos. Además, habría que añadir la necesaria calidad requerida a los metadatos que se encuentran asociados a estos conjuntos de datos para garantizar una recuperación precisa de los mismos, su confiabilidad y eficacia (Kluber y otros, 2018, p. 13).

Los portales de datos abiertos ofrecen a la ciudadanía un mejor acceso a la información y proporcionan un mecanismo de control del funcionamiento de las administraciones (Dorado Pérez, 2019 , p. 33). Según Royo-Montañés y BenítezGómez $(2019$, p. 2) son también un elemento clave para "poner a disposición de todos los agentes sociales los datos que posibilitan los tres objetivos perseguidos por el gobierno abierto (transparencia, participación y colaboración)".

Estos portales se ofrecen también como un instrumento de utilidad para facilitar el aprovechamiento de los datos públicos de la forma más efectiva posible, al poner en estos repositorios cientos de miles de conjuntos de datos a disposición de los ciudadanos en formatos que pueden ser procesables automáticamente (Cerrillo, 2014, p. 63).

Piedrabuena y Criado (2012, p. 10) señalan que "una estrategia de datos abiertos se vertebra en torno a un portal que organiza y permite acceder al catálogo de datos publicado por una organización". Los portales de datos abiertos son una herramienta básica y un escaparate idóneo para apreciar el desarrollo de las políticas de difusión de datos abiertos, ya que consiguen integrar y mantener importantes colecciones digitales de conjuntos de datos (Arquero Avilés, Marco Cuenca y Cobo Serrano, 2019, p. 254).

En nuestro territorio nacional existen un gran número de iniciativas de portales de datos abiertos que promueven el acceso, la difusión de los datos, su reutilización y aseguran la transparencia y la rendición de cuentas. Se trata de portales a nivel nacional, autonómico y local que liberan un gran número de conjuntos de datos donde podemos encontrar diferentes criterios a la hora de organizar y distribuir los mismos.

Este auge de iniciativas de portales abiertos, fomentada por la legislación nacional y europea en torno a la reutilización de la información pública y el gobierno abierto (1), viene a colisionar con una ausencia de criterios comunes y aceptados para evaluar estos portales. No obstante, existen algunos trabajos recientes que se han centrado en la evaluación de los portales, sus contenidos y características (Arquero Avilés y Marco Cuenca, 2014; Carrasco y Sobrepere, 2015; Medina Sánchez, 2015; Thorsby y otros, 2017, Zhu y Freeman, 2019: Correa, Souza y Correa da Silva, 2019), la evaluación de la usabilidad y acceso a la información (Manchova, Hub y Lnenicka, 2018; Santos, Galindo y Rover, 2019), la calidad de los datos abiertos (Manchova y Lnenicka, 2017; Abella, Ortiz de Urbina y De Pablos, 2018; Cadena-Vela, Fuster-Guilló y Mazón, 2019), el cumplimiento y reutilización de los datos (Vicente-Paños y Jordán-Alfonso, 2017; González-Límón y Rodríguez-Ramos, 2019), la transparencia y rendición de cuentas de los mismos (Curto Rodríguez, 2015; Lourenço, 2015; García García y Curto Rodríguez, 2019; Curto Rodríguez, 2020) o la aplicación y generación de modelos y metodologías para su análisis (Oviedo, Mazón y Zubcoff, 2018; Royo-Montañés y Benítez-Gómez, 2019).

Es indudable que vivimos en una verdadera revolución de los datos, la cantidad de datos que se generan aumenta constantemente y se acumulan a una velocidad sin precedentes. Muchas organizaciones están poniendo su foco en tecnologías emergentes como big data o machine learning (Artés, 2017, p. 21). Tanto los datos masivos, como el aprendizaje máquina o automático, necesitan datos de calidad para poder aprender y ofrecer información que pueda tener una incidencia real en la ayuda a las personas o servir de soporte para la toma de decisiones. Esta situación no es ajena al sector de la salud. La aparición de nuevas tecnologías y una conectividad mejorada han estimulado el crecimiento exponencial de los datos de salud (COCIR, 2019, p. 4).

Tal y como indica D'Agostino y otros $(2017$, p. 1) la disponibilidad de datos abiertos en el contexto de la salud fortalece el concepto de ciudadanos informados y ayuda a los pacientes, prestadores de servicios de salud e investigadores a tomar mejores decisiones, impulsar innovaciones, detectar brechas de conocimiento e identificar intervenciones más eficientes. Al mismo tiempo, la liberación de este tipo de datos permitirá utilizar a los gobiernos y las instituciones que forman parte de los sistemas de salud todo su potencial para generar políticas más efectivas, mejorar la calidad de la atención, disminuir costes en el cuidado de la salud y facilitar la decisión del paciente sobre los cuidados de su propia salud (D'Agostino y otros, 2017, p. 5)

\section{Objetivos y metodología}

La presente investigación tiene como objetivo principal estudiar las iniciativas de gobierno 
abierto en materia de publicación de datos en las diferentes Comunidades Autónomas españolas a través de sus portales de datos abiertos $\mathrm{y}$, en concreto, analizar el estado de publicación de los conjuntos de datos de salud en estos portales. Con ello, se pretende conocer el estado actual de apertura de datos a nivel autonómico, dado que las Administraciones Públicas Autonómicas son las principales gestoras de la mayor parte de servicios de carácter social dirigidos a los ciudadanos. Al mismo tiempo, se analizan los portales de datos abiertos de las diferentes Comunidades Autónomas con dos propósitos: el primero, conocer el estado actual de publicación de datos abiertos $y$, segundo, realizar una comparativa más detallada sobre el estado de apertura de los datos abiertos en salud.

Con respecto a la metodología, en cuanto a la localización y acceso a los portales autonómicos ha servido como base el portal oficial de Datos abiertos del Gobierno de España y su mapa de iniciativas de datos abiertos (2). A continuación, se accedió a todos los portales autonómicos para comprobar sus sistemas de clasificación y recuperación de conjuntos de datos. Con la excepción de las ciudades autónomas de Ceuta y Melilla, ya que no disponen de un portal de datos abiertos.

En la mayoría de los casos podían recuperarse los datos por una categoría relacionada con la salud o la sanidad. Para el caso de las Comunidades Autónomas de Cantabria y Galicia, se ejecutaron diferentes estrategias de búsqueda específicas, utilizando las palabras clave "salud" o "sanidad", adaptadas a sus buscadores para poder realizar una selección de los conjuntos de datos de interés. En el caso de Navarra, que también presentaba dificultades técnicas para la recogida de información, se intentó en un principio normalizar las temáticas ofrecidas en su catálogo con respecto a la Resolución de 19 de febrero de 2013, por la que se aprueba la Norma Técnica de Interoperabilidad (NTI) de recursos de la información, pero dado el grado de dificultad para poder establecer un criterio válido y adaptable, se descartó para el objeto de estudio.

Posteriormente, se seleccionaron un total de 10 indicadores que se organizaron por dominios, tomando como referencia para este tipo de organización la norma de calidad UNE 178301 (2015) de datos abiertos en ciudades inteligentes (3). De este modo, se crearon un total de cuatro dominios con el siguiente criterio: 1) dominio publicación de datos, que hace referencia a la disponibilidad y cantidad de conjuntos de datos publicados; 2) dominio de clasificación o categorización, relacionado con la categoría y subcategoría a la que pertenece el contenido de los conjuntos de datos; 3) dominio de reutilización, relacionado con los formatos disponibles para cada conjunto de datos; y, por último, 4) dominio de calidad, asociado al esquema de cinco estrellas de Tim Berners-Lee (2009) (4) (tabla I).

\begin{tabular}{|c|c|}
\hline Dominio & Indicador \\
\hline $\begin{array}{l}\text { Publicación } \\
\text { de datos }\end{array}$ & 1. Conjuntos de datos por CCAA \\
\hline $\begin{array}{l}\text { Clasificación o } \\
\text { categorización }\end{array}$ & $\begin{array}{l}\text { 2. Conjuntos de datos por sectores } \\
\text { 3. Conjuntos de datos de salud por } \\
\text { CCAA } \\
\text { 4. Conjuntos de datos de salud por } \\
\text { categorías } \\
\text { 5. Conjuntos de datos de salud } \\
\text { estadísticos por subcategorías }\end{array}$ \\
\hline Reutilización & $\begin{array}{l}\text { 6. Conjuntos de datos de salud por } \\
\text { formatos } \\
7 \text {. Conjuntos de datos de salud por } \\
\text { formatos reutilizables y no reutilizables } \\
8 \text {. Conjuntos de datos de salud por } \\
\text { formatos y CCAA }\end{array}$ \\
\hline Calidad & $\begin{array}{l}\text { 9. Conjuntos de datos de salud según } \\
\text { el esquema de las cinco estrellas de } \\
\text { Berners-Lee } \\
\text { 10. Conjunto de datos de salud según } \\
\text { el esquema de las cinco estrellas de } \\
\text { Berners-Lee por CCAA }\end{array}$ \\
\hline
\end{tabular}

Tabla I. Relación de tipos de dominios e indicadores

Posteriormente, se procedió a la recogida de datos de los portales, en el período comprendido entre el 15 de enero y el 1 de marzo de 2020. Los datos recogidos se relacionaban, principalmente, con: comunidad autónoma que publica los datos, categorías, subcategorías dentro de los conjuntos de datos de salud y formatos disponibles. Se consultó la misma información en todos los portales con objeto, posteriormente, de poder compararla. Los datos recabados se fueron volcando a una hoja de cálculo, organizando los mismos por tablas correspondientes para cada indicador. Los datos fueron revisados, clasificados y normalizados, para su posterior análisis mediante el uso de tablas dinámicas, tomando como referencia para ello la NTI sobre reutilización de recursos de la información (2013). La NTI se organiza en 22 sectores y cada sector puede incluir una o varias categorías.

\section{Resultados}

\subsection{Conjuntos de datos por Comunidades Autónomas}

Se han publicado un total de 19.166 conjuntos de datos por Comunidades Autónomas. En la figura 1 podemos observar que existe una importante diferencia entre la Comunidad Autónoma que más datos publica, el País Vasco (10.266), frente 
a la que menos pública, Extremadura (38). Solo las tres primeras Comunidades del ranking superan los 1.000 conjuntos publicados, alcanzando el $74 \%$ del total frente al $26 \%$ del resto.

\begin{tabular}{ll}
\hline CCAA & Conjuntos de Datos \\
\hline Pais Vasco & 10266 \\
\hline Aragón & 2849 \\
\hline Cantabria & 1039 \\
\hline Región de Murcia & 825 \\
\hline Andalucía & 677 \\
\hline Cataluña & 588 \\
\hline Castilla y León & 542 \\
\hline Castilla La Mancha & 473 \\
\hline Comunidad Valenciana & 455 \\
\hline Galicia & 392 \\
\hline La Rioja & 330 \\
\hline Canarias & 192 \\
\hline Comunidad de Madrid & $\mathbb{1} 181$ \\
\hline Islas Baleares & 169 \\
\hline Asturias & 150 \\
\hline Extremadura & 38 \\
\hline
\end{tabular}

Figura 1. Conjuntos de datos por Comunidades Autónomas

\begin{tabular}{|c|c|}
\hline Sectores & Conjuntos de datos \\
\hline Sector público & 2.549 \\
\hline Economía & 1.996 \\
\hline Demografía & 1.977 \\
\hline Medio Ambiente & 1.571 \\
\hline Sociedad y Bienestar & 1.529 \\
\hline Empleo & 1.035 \\
\hline Deporte & 1.004 \\
\hline Educación & 992 \\
\hline Cultura y ocio & 975 \\
\hline Hacienda & 954 \\
\hline Medio Rural y pesca & 763 \\
\hline Urbanismo e infraestructura & 746 \\
\hline Turismo & 566 \\
\hline Salud & 485 \\
\hline Vivienda & 399 \\
\hline Ciencia y tecnología & 314 \\
\hline Transporte & 289 \\
\hline Comercio & 241 \\
\hline Legislación y justicia & 234 \\
\hline Industria & 214 \\
\hline Seguridad & 179 \\
\hline Energía & 154 \\
\hline
\end{tabular}

Figura 2. Conjuntos de datos por sectores

\subsection{Conjuntos de datos por sectores}

Con relación al total de conjuntos de datos por sectores podemos advertir que existen tres temas que despuntan con respecto al resto y son: sector público con 2.549 conjuntos de datos (el
$13,3 \%$ del total), economía con 1.996 conjuntos $(10,4 \%)$ y demografía con $1.977(10,3 \%)$ (figura $2)$. Con respecto a los datos de salud se corresponden con 485 conjuntos de datos (un $2,5 \%$ del total) y se sitúan en el puesto 14 de un ranking de 22 categorías (figura 3 ).

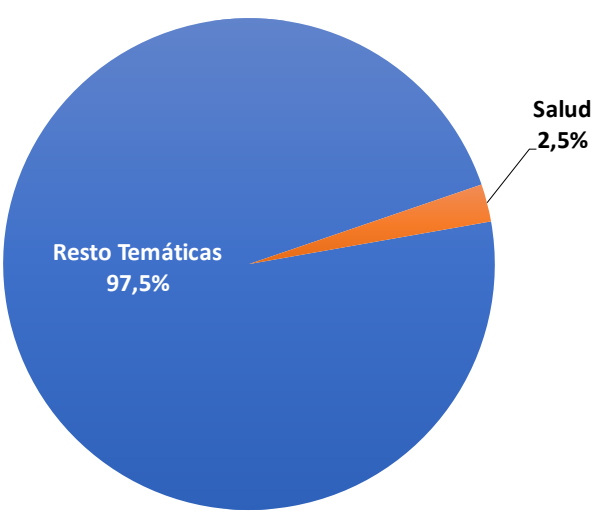

Figura 3. Conjuntos de datos de salud frente al resto de sectores (en \%)

\begin{tabular}{ll}
\hline CCAA & Conjuntos de datos de salud \\
\hline Aragón & 124 \\
\hline Pais Vasco & 116 \\
\hline Región de Murcia & 63 \\
\hline Castilla La Mancha & 39 \\
\hline Castilla y León & 34 \\
\hline Cataluña & 21 \\
\hline Cantabria & 16 \\
\hline Comunidad Valenciana & 12 \\
\hline La Rioja & 12 \\
\hline Asturias & 11 \\
\hline Comunidad de Madrid & 11 \\
\hline Galicia & 9 \\
\hline Andalucía & 7 \\
\hline Islas Baleares & 6 \\
\hline Canarias & 2 \\
\hline Extremadura & 2 \\
\hline
\end{tabular}

Figura 4. Conjuntos de datos de salud por Comunidades Autónomas

3.3. Conjuntos de datos de salud por Comunidades Autónomas

El siguiente indicador viene determinado por la disponibilidad y cantidad de conjuntos de datos publicados sobre salud por Comunidad Autónoma. Teniendo en cuenta los 485 conjuntos de datos de salud, destaca Aragón como la Comunidad Autónoma que mayor número de conjuntos de datos de salud ha publicado con un total de 124 conjuntos $(25,6 \%)$ y en una segunda posición se encuentra el País Vasco con 116 conjuntos (24\%) (figura 4, arriba). 


\subsection{Conjuntos de datos de salud por categorías}

Con relación a las categorías o materias, dentro del sector de la salud, podemos encontrar que principalmente predominan las relacionadas con las estadísticas sanitarias con un total de 245 conjuntos de datos $(50,5 \%$ del total) (figura 5$)$.

\begin{tabular}{|c|c|}
\hline Categorías & Conjuntos de datos \\
\hline Estadísticas & 245 \\
\hline Salud y medioambiente & 70 \\
\hline Instalaciones sanitarias & 50 \\
\hline Miscelánea & 32 \\
\hline Registros & 29 \\
\hline Gastos/presupuestos & 18 \\
\hline Prestaciones sanitarias & 13 \\
\hline Ámbito farmacológico & 10 \\
\hline Prevención & 6 \\
\hline Encuestas & 6 \\
\hline CIE-10 & 6 \\
\hline
\end{tabular}

Figura 5. Conjuntos de datos de salud por categorías

3.5. Conjuntos de datos de salud estadísticos por subcategorías

Dentro de los conjuntos de datos predominantes, los relacionados con las estadísticas sanitarias, destacan aquellos que tienen que ver con: la mortalidad (44 conjuntos, 18\%), discapacidad (40 conjuntos, $16,3 \%$ ), indicadores sociales (35 conjuntos, 14,3\%), limitaciones (28 conjuntos, $11,4 \%$ ), sanidad pública (23 conjuntos, $9,4 \%$ ), etc. (figura 6 ).

\begin{tabular}{ll}
\hline Estadísticas & Conjuntos de datos \\
\hline Mortalidad & 44 \\
\hline Discapacidad & 40 \\
\hline Indicadores sociales & 35 \\
\hline Limitaciones & 28 \\
\hline Sanidad publica & 23 \\
\hline Interrupción voluntaria del embarazo & 18 \\
\hline Personal sanitario & 18 \\
\hline Recursos & 10 \\
\hline Enfermedades de cumplimiento obligatorio & 9 \\
\hline Listas de espera & 7 \\
\hline Enfermedades crónicas & 5 \\
\hline Actividad asistencial & 4 \\
\hline Donaciones & 3 \\
\hline Morbilidad & 1 \\
\hline
\end{tabular}

Figura 6. Conjuntos de datos de salud estadísticos por subcategorías

\subsection{Conjuntos de datos de salud por formatos}

Respecto a este indicador hay que señalar que, generalmente, un mismo conjunto de datos puede haber sido publicado en varios formatos, por lo que el total de conjuntos de datos no va a coincidir con el total de formatos publicados.

\begin{tabular}{ll}
\hline Conjuntos de datos publicados en salud & 485 \\
\hline $\begin{array}{l}\text { Conjuntos de datos publicados en salud } \\
\text { según los formatos utilizados }\end{array}$ & 798 \\
\hline
\end{tabular}

Tabla 2. Publicación total de conjuntos de datos en salud frente a formatos

Atendiendo al tipo de formato utilizado en la publicación de datos sanitarios podemos observar que de las dieciséis comunidades autónomas analizadas, trece de ellas están publicando datos en el formato abierto CSV lo que supone un $40 \%$ del total de los formatos utilizados (317 conjuntos). Nueve comunidades publican en el formato propietario XLS/XLSX (200 conjuntos, $25 \%$ ) y en formato JSON/geoJSON (78 conjuntos, $10 \%$ ) y ocho en formato XML (68 conjuntos, $9 \%$ ) (figura 7).

\begin{tabular}{ll|l}
\hline Formatos & Total conjuntos de datos & CCAA \\
\hline CSV & 317 & 13 \\
\hline XLS/XLSX & 200 & 9 \\
\hline JSON/geoJSON & 78 & 9 \\
\hline XML & 68 & 8 \\
\hline HTML & 38 & 4 \\
\hline RDF & 35 & 4 \\
\hline RSS & 19 & 3 \\
\hline PDF & 18 & 3 \\
\hline KML & 7 & 3 \\
\hline ODS & 7 & 2 \\
\hline SHP & 6 & 3 \\
\hline ZIP & 3 & 2 \\
\hline WMS & 1 & 1 \\
\hline WMF & 1 & 1 \\
\hline
\end{tabular}

Figura 7. Conjuntos de datos por formatos y CCAA que los usan

Un total de 739 conjuntos de datos son reutilizables frente a los 59 conjuntos de datos no reutilizables (html, pdf y zip) (figura 8).

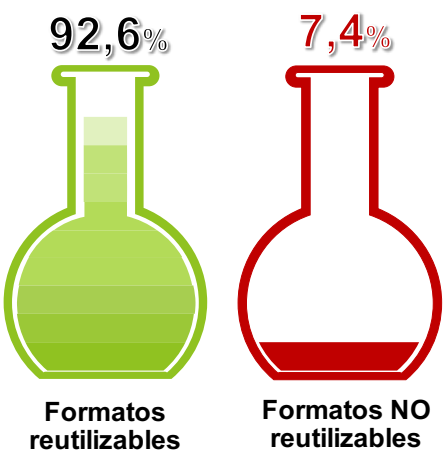

Figura 8. Conjuntos de datos en salud por formatos reutilizables y no reutilizables 
Un total de 5 Comunidades Autónomas ofrecen conjuntos no reutilizables y 10 ofrecen siempre conjuntos reutilizables. Solamente Asturias ofrece sus conjuntos de datos en un único formato reutilizable (xml) (tabla 3 ).

\begin{tabular}{|c|c|}
\hline$\star$ & $7,4 \%$ \\
\hline 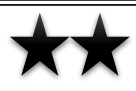 & $25,8 \%$ \\
\hline & \\
\hline
\end{tabular}

Figura 9. Conjuntos de datos en salud según esquema de las cinco estrellas de Berners-Lee

\begin{tabular}{|c|c|c|c|c|}
\hline Comunidad & $\star$ & $\star \star$ & $\star \star \star$ & $\star \star \star \star$ \\
\hline Andalucía & $\begin{array}{l}\text { PDF, } \\
\text { HTML }\end{array}$ & XLS & $\begin{array}{l}\text { CSV, XML, } \\
\text { ODS, } \\
\text { JSON, }\end{array}$ & \\
\hline Aragón & & XLS & CsV & \\
\hline Asturias & & & XML & \\
\hline Islas Baleares & & XLS & $\begin{array}{l}\text { CSV, KML, } \\
\text { RSS, XML, } \\
\text { JSON }\end{array}$ & RDF \\
\hline Canarias & & SHP & CsV & \\
\hline Cantabria & HTML & XLS & JSON & RDF \\
\hline $\begin{array}{l}\text { Castilla-La } \\
\text { Mancha }\end{array}$ & $\begin{array}{l}\text { PDF, } \\
\text { HTML }\end{array}$ & XLS & $\begin{array}{l}\text { CSV, ODS, } \\
\text { JSON }\end{array}$ & \\
\hline Castilla y León & & & CSV, XML & \\
\hline Cataluña & $\begin{array}{l}\text { PDF, } \\
\text { ZIP }\end{array}$ & $\begin{array}{l}\text { XLS, } \\
\text { SHP }\end{array}$ & $\begin{array}{l}\text { CSV, RSS, } \\
\text { XML, KML, } \\
\text { JSON }\end{array}$ & RDF \\
\hline $\begin{array}{l}\text { Com. } \\
\text { Valenciana }\end{array}$ & & & CSV & \\
\hline Extremadura & & XLS & CSV & \\
\hline Galicia & $\begin{array}{l}\text { HTML, } \\
\text { ZIP }\end{array}$ & & $\begin{array}{l}\text { KML, } \\
\text { WMS, WFS, } \\
\text { RSS }\end{array}$ & \\
\hline La Rioja & & XLS & $\begin{array}{l}\text { CSV, XML, } \\
\text { JSON }\end{array}$ & \\
\hline $\begin{array}{l}\text { Comunidad de } \\
\text { Madrid }\end{array}$ & & & CSV, JSON & \\
\hline $\begin{array}{l}\text { Región de } \\
\text { Murcia }\end{array}$ & & XLS & $\begin{array}{l}\text { CSV, JSON, } \\
\text { XML }\end{array}$ & RDF \\
\hline País Vasco & & XLS & $\begin{array}{l}\text { CSV, XML, } \\
\text { JSON }\end{array}$ & \\
\hline
\end{tabular}

Tabla 3. Formatos por Comunidades Autónomas según las cinco estrellas de Berners-Lee

Si examinamos estos datos en el esquema de las cinco estrellas de Berners-Lee (2009) podemos comprobar que formatos de 1 estrella, no reutilizables (pdf, html y zip), existen un total de 59 conjuntos $(7,4 \%$ del total); formatos de 2 estrellas, reutilizables propietarios (xls/xlsx, shp) hay 206 conjuntos (25,8\%); de 3 estrellas, formatos reutilizables no propietarios (csv, $\mathrm{xml}$, json, ods, $\mathrm{kml}$, rss, wms, wmf) ascienden a los 498 conjuntos $(62,4 \%)$; los conjuntos de 4 estrellas que utilizan estándares abiertos W3C (rdf, sparql) son $35(4,4 \%)$ y conjuntos de 5 estrellas, que enlazan datos con otros datos para proveer contexto (linked open data) no se han encontrado (figura 9).

Un total de 5 Comunidades Autónomas tienen datos en formatos 1 estrella, 11 Comunidades en formatos de 2 estrellas, 16 Comunidades mantienen formatos de 3 estrellas, reutilizables y en formato no propietario, y, por último, 4 Comunidades contienen en sus portales formatos 4 estreIlas (tabla 3).

\section{Conclusiones}

Los portales de las Comunidades Autónomas recogen registros de todo tipo: información medioambiental, demográfica, social, cultural, etc. Todas las Comunidades Autónomas coinciden en el hecho de recoger datos de salud y, dado que es un sector que preocupa especialmente a la sociedad, puede considerarse un gran avance. Sin embargo, el número de conjuntos de datos sobre salud publicados por las administraciones autonómicas pueden aún considerarse escasos, ya que solamente representan un $2,5 \%$ del total.

El análisis de los datos de salud viene a indicarnos que existen unas Comunidades Autónomas más avanzadas que otras en la publicación de este tipo de datos. Aragón y País Vasco se encuentran a la cabeza con más de 100 conjuntos de datos cada una y, por el contrario, Galicia, Andalucía, Baleares, Canarias y Extremadura son las más rezagadas, sumando entre todas ellas un total de 26 conjuntos de datos. Se infiere, por tanto, una doble velocidad en la apertura de este tipo de datos.

En lo que respecta a los formatos utilizados para la publicación de estos conjuntos de datos sanitarios, algunas administraciones siguen utilizando formatos no reutilizables; aunque cada vez en menor medida. La tendencia de dicha práctica es que sea totalmente reducida a favor de la utilización de formatos más avanzados para la apertura de datos.

Se puede observar una clara disposición por parte de las Comunidades Autónomas a publicar en formatos nivel 3 estrellas, es decir, datos en formatos estructurados, abiertos y no propietarios. Es frecuente también la utilización de formatos de 2 estrellas, formatos estructurados 
(legibles por máquinas) que facilitan su tratamiento pero que se encuentran en un formato propietario, lo que puede exigir la compra de alguna licencia de software para poder trabajar con la información. La práctica más habitual entre Comunidades Autónomas es la publicación de conjuntos de datos en varios formatos $y$, generalmente, en formatos de nivel 2 y 3 estrellas. No obstante, quedan Comunidades Autónomas que siguen usando formatos de nivel 1 , formatos no estructurados que requieren de la aplicación de técnicas y esfuerzo extra, y que, por lo tanto, no son recomendables utilizar.

De cara a un futuro, el reto puede estar en aumentar el número de conjuntos de datos de calidad puestos a disposición de los posibles usuarios e incentivar a las organizaciones del sector de la salud para su publicación con el objetivo de generar valor social y económico. Estos datos pueden ser realmente útiles para ser utilizados en sistemas de datos masivos o de aprendizaje automático para la toma de decisiones clínicas y epidemiológicas, además de ser un factor clave para generar herramientas preventivas o aplicaciones de salud que fomenten el autocuidado y permitan a los pacientes tener un mayor control sobre su salud.

\section{Notas}

(1) En concreto, nos referimos a la Directiva 2013/37/UE relativa a la reutilización de la información en el sector público que establece políticas de apertura a la información, propiciando la disponibilidad y la reutilización de la información en el sector público con fines privados o comerciales, con restricciones mínimas o nulas de carácter jurídico, técnico y económico, buscando favorecer la circulación de la información no solo para los agentes económicos, sino también entre el público en general. En el plano nacional, podemos encontrar la Ley 37/2007, de 16 de noviembre, sobre reutilización de la información del sector público, considerada realmente como la transposición al ordenamiento jurídico español de la directiva 2003/98/UE. Esta Ley se desarrolla a través del Real Decreto 1495/2011, de 24 de octubre, sobre reutilización de la información del sector público, para el ámbito del sector público estatal. Del mismo modo hay que destacar la Ley 19/2013 de transparencia, acceso a la información pública y buen gobierno que se centra en el desarrollo de una adecuada cultura para la transparencia y la rendición de cuentas de las administraciones públicas a todos sus niveles y donde se recomienda que todo portal de transparencia tenga asociado un portal de datos abiertos. Además, a nivel normativo, las Comunidades Autónomas también han elaborado sus propias leyes relacionadas con el gobierno abierto.

(2) El portal de datos abiertos del Gobierno de España se encuentra disponible en la siguiente dirección de Internet: http://datos.gob.es. Este portal cuenta en su página principal con el Mapa de Iniciativas de Datos Abiertos que incluye, en el momento de su consulta (1.02.2020), un total de 299 iniciativas, de las que 18 se corresponden con la Administración Autonómica, 43 con la Administración del Estado, 229 con la Administración local y 9 con las Universidades (Datos.gob.es, 2020).
(3) La norma UNE 178301 establece una colección de métricas que tienen en consideración aspectos que precisan ser contemplados en las iniciativas de datos abiertos. Estas métricas se encuentran organizadas en forma de dominios y dimensiones para facilitar su estructuración y comprensión.

(4) El esquema de datos de las cinco estrellas de Tim Berners-Lee es un sistema piramidal de asignación de estrellas para determinar el grado de disponibilidad y accesibilidad de los formatos de los conjuntos de datos abiertos, con objeto de poder medir la calidad de los datos abiertos en base a su posible nivel de reutilización (Arquero Avilés y Marco Cuenca, 2014, p. 103).

\section{Referencias}

Abella, Alberto, Ortiz de Urbina, Marta; De Pablos, Carmen (2018). Indicadores de calidad de los datos abiertos: el caso del portal de datos abiertos de Barcelona. // El Profesional de la Información. 27:2. 375-382.

Arquero Avilés, Rosario; Marco Cuenca, Gonzalo (2014). El Portal de datos abiertos de la Unión europea: análisis y evaluación. // Revista General de Información y Documentación. 24:1. 99-118.

Arquero Avilés, Rosario; Marco Cuenca, Gonzalo y Cobo Serrano, Silvia (2019). Datos abiertos sobre salud: portales y recursos de información para su reutilización. // En: Montesi, Michela; Marco Cuenca, Gonzalo y Ramírez Martín, Susana María. Información, Salud y ciudadanía. Gijón: Trea, 2019.

Artés, Antonio (2017). Tecnologías emergentes en el tratamiento de datos. // I+S. Informática y Salud. 123 (junio). 21-25.

Berners-Lee, Tim (2009). Linked Data. // https://www.w3.org/Designlssues/LinkedData.html (1-022020).

Cadena-Vela, Susana; Fuster-Guilló, Andrés; Mazón, José Norberto (2019). Publicando datos abiertos considerando criterios de calidad. // RISTI. Revista Ibérica de Sistemas e Tecnologias de Informaçao. E22. 295-308.

Carrasco, Carlos; Sobrepere, Xavier (2015). Open government data: An assessment of the Spanish municipal situation. // Social Science Computer Review. 33:5. 631644.

Cerrillo, Agustí (2014). Los principios de datos abiertos en la legislación española. // Revista de Internet, Derecho y Política. 19 (oct). 62-77.

COCIR (2019). European Health Data Space. Towards a better patient outcome. // Bruselas: COCIR, 2019. https://www.cocir.org/media-centre/publications/article/european-health-data space-towards-a-better-patient-outcome.html (14-02-2020)

Correa, Andreiwid Sheffer; Souza, Raul Mendes de; Correa da Silva, Flavio Soares (2018). Towards an automated method to assess data portals in the deep web. // Government Information Quarterly. 36:3. 412-426.

Curto Rodríguez, Ricardo (2015). Los portales de datos autonómicos y la rendición de cuentas. // Auditoría pública. 66. 75-83.

Curto Rodríguez, Ricardo (2020). Transparencia operativa de las Comunidades Autónomas españolas mediante sus portales de datos abiertos. // El Profesional de la Información. 29:1.

D’Agostino, Marcelo; Martí, Myrna; Mejía, Felipe y otros (2017). Estrategia para la gobernanza de datos abiertos en salud: un cambio de paradigma en los sistemas de información. // Revista Panamericana de Salud Pública. 41. $1-6$. 
Datos.gob.es (2020). Mapa de Iniciativas de datos abiertos. // Madrid: Ministerio de Economía y Empresa; Red.es. https://datos.gob.es/es/iniciativas (3-02-2020)

Dorado Pérez, Esperanza (2019). Portales de datos abiertos. Experiencia práctica en el Consejo de Transparencia y Protección de Datos de Andalucía. // Revista Española de Transparencia. 9. 33-38.

García García, Jesús; Curto Rodríguez, Ricardo (2019). El ejercicio de la rendición de cuentas mediante portales de datos abiertos en las comunidades autónomas españolas. // Revista de Internet, Derecho y Política. 29 (marsep). 1-15.

González-Limón, Myriam; Rodríguez-Ramos, Asunción (2019). Revisión del cumplimiento de los Datos Abiertos por los Ayuntamientos españoles en la iniciativa Aporta. // Revista Española de Documentación Científica. 42:4 (oct-dic). e247.

Kluber, Sylvian; Robert, Jerermy; Neumaier, Sebastian y otros (2018). Comparision of metadata quality in open data portals using de analytic hierarchy process. // Government Information Quarterly. 35:1. 13-29.

Lourenço, Rui-Pedro (2015). An analysis of open government portals: A perspective of transparency. // Government Information Quarterly. 32:1. 323-332.

Manchova, Renata; Hub, Miloslav; Lnenicka, Martin (2018). Usability evaluation of open data portals Evaluating data discoverability, accessibility, and reusability from a stakeholders' perspective. // ASLIB. Journal of Information Management. 70:3. 252-268.

Manchova, Renata; Lnenicka, Martin (2017). Evaluating the quality of Open Data Portas on the National Level. // Journal of Theoretical and Applied Electronic Commerce Research. 12:1. 21-41.

Medina, Vanessa (2015). Análisis de proyectos open data en España: propuestas de mejora. // Cuadernos de gestión de información. 5:1. 53-72.

Oviedo, Edgard; Mazón, José Norberto; Zubcoff, José Jacobo (2018). Modelo de calidad y madurez para portales de datos abiertos. // CONICYT. Boletín de Ciencia y Tecnología. 191 (octubre). 1-6.
Paños-Vicente, Adrián; Jordán-Alfonso, Aurea (2017). Acceso a la información pública y su reutilización en las comunidades autónomas: evaluación de la reutilización de datos abiertos. // El Profesional de la Información. 26:3. 381-391.

Piedrabuena, Agustina; Criado, Luis (2012). Opendata, oportunidad escondida y semilla de la Web semántica. // RUIDERAe: Revista de Unidades de Información. 2. 1-21.

Pintos, Carlos; Marín, José Luis (2013). Open data. Reutilización de la información pública. // La Coruña: NetBiblo; Madrid: INAP, 2013

Resolución de 19 de febrero de 2013, de la Secretaría de Estado de Administraciones Públicas, por la que se aprueba la Norma Técnica de Interoperabilidad de Reutilización de recursos de la información. // Boletín Oficial del Estado. 54 (4 de marzo de 2013) 17045-17071.

Royo-Montañés, Sonia; Benítez-Gómez, Alberto (2019). Portales de datos abiertos. Metodología de análisis y aplicación a municipios españoles. // El Profesional de la Información. 28:6.1-13.

Sadiq, Shazia; Indulska, Marta (2017). Open data: quality over quantity. // International Journal of Information Maganement. 37:3. 150-154.

Santos, Paloma María; Galindo, Fernando; Rover, Aires José (2019). Acceso democrático a la información en los portales del gobierno español: un análisis comparativo entre los años 2013 y 2015. // Ibersid. 13:2 (jul-dic).13-20.

Thorsby, Jeffery; Stowers, Genie NL, Wolsegel, Kristen; Tumbuan, Ellie (2017). Understanding the content and features of open data portals in American cities. // Government Information Quaterly. 34:1. 53-61.

UNE 178301 (2015). Ciudades inteligentes. Datos abiertos (Open data). // Madrid: AENOR, 2015.

Zhu, Xiaohua; Freeman, Mark Antony (2019). An evaluation of US municipal open data portals: A user interaction framework. // Journal of the Association for Information Science and Technology. 70:1. 27-37.

Enviado: 2020-03-25. Segunda versión: 2020-05-16. Aceptado: 2020-06-04.

Bertos Quesada, María Ángeles; Marco Cuenca, Gonzalo. Análisis del estado actual de apertura de datos en salud a nivel autonómico a través de los portales de datos abiertos. // Ibersid. 14:1 (en.-jun. 2020) 87-94. ISSN 1888-0967. 